\title{
Avaliação de cultivares de girassol em savana de Roraima
}

\author{
Oscar José SMIDERLE ${ }^{1}$; Moisés MOURÃO JR²; Daniel GIANLUPPI ${ }^{2}$
}

\begin{abstract}
RESUMO
A cultura do girassol (Helianthus annuus L.) pode ser conduzida em diversas épocas do ano, destacando-se desta forma, entre as culturas viáveis de serem exploradas nas savanas (lavrados) de Roraima. Entretanto, as cultivares podem apresentar diferenças de adaptação e desenvolvimento dependendo da área de cultivo. Assim, desenvolveu-se este estudo comparativo em área experimental da Embrapa Roraima em 2000, visando avaliar o desempenho de seis cultivares (Agrobel 910; Agrobel 920; Cargill 11; Embrapa 122; Morgan 742; e Rumbosol 91) semeadas em duas épocas (seca, com irrigação suplementar; e chuvosa) em Boa Vista, Roraima. Utilizaram-se parcelas subdivididas num delineamento de blocos ao acaso com quatro repetições, sendo as duas épocas de semeadura em 20 de janeiro e 19 de julho. As subparcelas constaram de quatro fileiras de $6 \mathrm{~m}$, distanciadas de $0,90 \mathrm{~m}$, sendo $0,30 \mathrm{~m}$ o espaçamento entre plantas. As cultivares Cargill 11, Rumbosol $91 \mathrm{e}$ Agrobel 910 foram as mais produtivas para cultivo nas condições climáticas das savanas de Roraima, e a semeadura em janeiro é mais indicada, comparada a de julho.
\end{abstract}

\section{PALAVRAS CHAVES}

emergência; cultivares; Helianthus annuus

\section{Avaliation of Sunflower Cultivars in Savanna Ecosystem of Roraima.}

\begin{abstract}
Sunflower (Helianthus annuus L.) can be cultivated at different times of the year. Hence, this oilseed crop stands out among the viable crops to be explored in the savanna area of Roraima. However, cultivars can perform differently depending on the region they are grown. Accounting on this, a study was carried out to evaluate the effect of sowing time for this crop in Boa Vista, Roraima, utilizing six cultivars (Agrobel 910; Agrobel 920; Cargill 11; Embrapa 122; Morgan 742; and Rumbosol 91) in a split-plot scheme, in a randomized complete blocks design, with two plots (sowing time: January 20, with supplementation of water, and July 19). The sub-plots consisted of four rows, measuring 6 meach, distanciated $0.90 \mathrm{~m}$; the distance between plants inside the row was $0,30 \mathrm{~m}$. The cultivars cargill 11, rumbosol 91 and agrobel 910 were the most productive for cultivation in the savanna ecosystem in Roraima. The sowing of sunflower in January was more appropriate than July.
\end{abstract}

\section{KEYWORDS}

Swing time; cultivars; Helianthus annuus

\footnotetext{
${ }^{1}$ Eng.Agr. DSc. Pesquisador Embrapa Roraima. CP.133, CEP 69301-970. E-mail: ojsmider@cpafrr.embrapa.br

${ }^{2}$ MSc. Pesquisador Embrapa Roraima. C.P.133, CEP69301-970. E-mail: mmourao@cpafrr.embrapa.br
} 


\section{INTRODUÇÃO}

A cultura do girassol, em fase de implantação em Boa Vista, apresenta-se como uma opção promissora para a agricultura de Roraima. Dentre as oleaginosas é uma cultura que possui um dos maiores índices de crescimento no mundo, ocupando o segundo posto como fonte de óleo vegetal comestível, e o quarto, como fonte de proteínas vegetais (Reyes et al., 1985). O interesse que o girassol está despertando deve-se a qualidade e ao múltiplo uso de seus produtos derivados e ainda à sua ampla adaptabilidade, podendo se constituir numa alternativa adicional para cultivo e, principalmente, compor um sistema de produção de grãos, com grande potencial de utilização (Endres, 1993).

Dentre os óleos vegetais, o óleo de girassol destaca-se por suas excelentes características físico-químicas e nutricionais. Possui alta relação de ácidos graxos poliinsaturados (65,3\%)/ saturados $(11,6 \%)$, sendo que o teor de poliinsaturados é constituído, em grande parte, pelo ácido linoléico (65\%). Este é essencial ao desempenho das funções fisiológicas do organismo humano e deve ser ingerido através dos alimentos, já que nãoé sintetizado pelo organismo. Por essas características, é um dos óleos vegetais de melhor qualidade nutricional e organoléptica do mundo (Castro et al., 1997).

Existem ainda espaços no sistema agrícola utilizado nas savanas de Roraima que podem ser ocupados pelo girassol. Há que se definir, no entanto, as características desses espaços, para possibilitar uma exploração racional e a diversificação do sistema produtivo.

A produção e a pesquisa de girassol no Brasil abrangem diferentes regiões com condições edafoclimáticas distintas. Essas atividades requerem que as observações, avaliações e sugestões a respeito da tecnologia de produção a ser adotada sejam realizadas durante todas as fases de desenvolvimento da planta.

Aépoca de semeadura é de importância fundamental para se obter sucesso no cultivo do girassol, sendo bastante variável e dependente das características climáticas de cada região de cultivo (Castro et al., 1997). A cultura pode ser semeada durante o ano todo, caso haja disponibilidade de água. Entretanto, para Reyes et al. (1985) o principal fator de sucesso da cultura é a época de semeadura.

A duração do período de crescimento vegetativo depende, principalmente, do genótipo, da temperatura e da disponibilidade de água. O período inicial de crescimento é lento. Até o início do florescimento as plantas atingem 90-95\% da altura total. A fase de emergência de plântulas requer sementes de qualidade para ocorrer, no máximo, em cinco ou sete dias após a semeadura. A profundidade maior que $5 \mathrm{~cm}$ e chuvas torrenciais ou ausência de água na camada de 10 a 15 $\mathrm{cm}$ de solo, podem prorrogar o período para até 15 dias, ocasionando enfraquecimento das plântulas, baixo estande e atraso na fase inicial de crescimento. Problemas que ocorrem na emergência das plântulas, resultarão em desuniformidade no desenvolvimento das plantas e na população de plantas, as quais permanecem até a colheita (Castiglioni et al., 1997).
$\mathrm{Na}$ condução do cultivo do girassol, é importante o conhecimento da fenologia da planta em cada fase do desenvolvimento da cultura. Da emergência aos 30 dias (aparecimento do botão floral), o crescimento é lento, consumindo pouca água e nutrientes. A partir desse período até o final do florescimento, o crescimento é rápido, aumentando o consumo de água e de nutrientes (Castro et al., 1997).

Essa oleaginosa tem, pelas suas características peculiares, possibilidade de adaptação a extensas áreas do país, podendo ser cultivada em semeaduras de verão e de outono (Embrapa, 1983; Costa et al., 2000). Em cultivo de sucessão da cultura principal, o girassol poderá ser encontrado, em futuro próximo, vegetando em extensas áreas, que no momento estão ociosas a espera de boas opções de plantio. Desse modo, a oleaginosa apresenta-se como um cultivo potencial para o Estado de Roraima, com possibilidade de semeaduras durante um longo período do ano, restando definir apenas as épocas e as cultivares mais apropriadas.

O rendimento obtido pelo girassol depende da cultivar assim como das condições ambientais a que for submetida (Embrapa, 1999; Abreu et al., 2001). No Brasil grande parte territorial é considerada apta para o cultivo do girassol, por apresentar condições climáticas favoráveis ao seu desenvolvimento. Em Roraima a informação a cerca de produtividade ou de desenvolvimento de plantas da cultura está em fase inicial com abrangência apenas experimental.

Pelo exposto, em Roraima, existe ainda carência de resultados que permitam indicar, com maior segurança, híbridos e/ou cultivares de girassol aos agricultores. Portanto, neste trabalho, objetivou-se avaliar a adaptabilidade de seis cultivares de girassol semeadas em duas épocas, nas condições edafoclimáticas de savanas em Boa Vista - RR.

\section{MATERIAL E MÉTODOS}

Foram utilizadas sementes de seis cultivares de girassol (Agrobel 910; Agrobel 920; Cargill 11; Embrapa 122; Morgan 742; e Rumbosol 91) obtidas junto a produtores, comerciante de sementes de girassol e Embrapa Soja. O trabalho foi realizado no campo experimental do Monte Cristo, pertencente a Embrapa Roraima, com utilização de pivô central para a suplementação de água.

O experimento foi instalado obedecendo delineamento estatístico fatorial, com quatro repetições, sendo as duas épocas de semeadura ( $2^{\mathrm{a}}$ quinzena de janeiro e $2^{\mathrm{a}}$ quinzena de julho de 2000), as parcelas principais, e as seis cultivares as subparcelas, compostas por quatro fileiras de $6 \mathrm{~m}$ de comprimento, em $0,90 \mathrm{~m}$ entre linhas e $0,30 \mathrm{~m}$ de espaçamento entre plantas. As duas fileiras centrais foram utilizadas como área útil $\left(10,8 \mathrm{~m}^{2}\right)$.

O preparo do solo foi realizado com uma aração profunda seguida de duas gradagens (Bolson, 1990) deixando o solo pronto, para proporcionar a emergência uniforme e desenvolvimento satisfatório das plantas de acordo com as 


\section{ACTA AMAZONICA}

características da cultura. O solo para o cultivo apresentava as seguintes características: $\mathrm{pH}$ (índice $\mathrm{SMP}$ ) $=6,8 ; \mathrm{Al}$ trocável $\left(\mathrm{cmol}_{\mathrm{c}} \cdot \mathrm{dm}^{-3}\right)=0,01 ; \mathrm{Ca}+\mathrm{Mg}\left(\mathrm{cmol}_{\mathrm{c}} \cdot \mathrm{dm}^{-3}\right)=3,85 ; \mathrm{P}_{2} \mathrm{O}_{5}$ $\left(\mathrm{mg} \cdot \mathrm{dm}^{-3}\right)=180,36 ; \mathrm{K}_{2} \mathrm{O}\left(\mathrm{mg} \cdot \mathrm{dm}^{-3}\right)=129,48 ;$ Matéria orgânica $=20,2 \mathrm{~g} \cdot \mathrm{dm}^{-3}$, e textura com $29,72 \%$ de argila e $14,11 \%$ de silte.

A adubação de semeadura foi realizada manualmente aplicando-se $400 \mathrm{~kg} \cdot \mathrm{ha}^{-1}$ da fórmula 2-20-20 de NPK, e em cobertura $100 \mathrm{~kg} \cdot \mathrm{ha}^{-1}$ de uréia, além de $12 \mathrm{~kg} . \mathrm{ha}^{-1}$ de Bórax (11,5\% de B). A semeadura foi realizada com duas sementes a cada 0,30 m de sulco. O desbaste, foi realizado aos 12 dias após a emergência, quando se deixou apenas uma planta por "cova" e aproximadamente 41.000 plantas.ha ${ }^{-1}$.

Os parâmetros avaliados foram: porcentagem de emergência, avaliada aos 12 dias após a emergência, com contagens a cada dois dias; velocidade de emergência de plântulas, conforme Popinigis (1985); altura de plantas, tomando como medida a inserção do capítulo até o colo da planta, no florescimento pleno, $\mathrm{R}_{55}$ (Schneiter \& Miller, 1981); diâmetro da haste, no final do florescimento pleno, medindose com paquímetro 20 plantas a $0,05 \mathrm{~m}$ do nível do solo; diâmetro de capítulos, medindo-se 30 capítulos da área útil no momento da colheita; ciclo, número de dias da emergência até a maturidade fisiológica; e, a produtividade, pela pesagem dos aquênios produzidos na área útil e corrigidos para $11 \%$ de umidade e para um hectare, além dos teores médios de óleo para cada cultivar nos dois cultivos.

As unidades de medida e as abreviaturas adotadas para as variáveis avaliadas foram: duração do ciclo (CIC) em dias; altura das plantas (ALT); diâmetro da haste (DHAS) e diâmetro do capítulo (DCAP) em centímetro; velocidade de emergência (VEMG) apresentada em índice; emergência (EMG) em percentagem e a produtividade (PROD) em kg.ha-1 .

O modelo de análise adotado considerou os efeitos da (E) época de cultivo e (V) de cultivares, bem como a interação entre os efeitos $(\mathrm{E} * \mathrm{~V})$, sendo estes definidos por meio de análise de variância do tipo fatorial. Componentes de variância para todas as variáveis agronômicas tomadas foram obtidos através do método de esperança de quadrados médios (EQM). Os valores médios foram ordenados segundo o teste de Tukey. Tanto no teste de ordenção de médias, quanto na análise de variância o nível de significância adotado foi o de 5\%.

\section{RESULTADOS E DISCUSSÃO}

As sementes das cultivares de girassol utilizadas apresentavam poder germinativo superior a $86 \%$. O efeito da época de plantio (E) foi significante em todas as variáveis agronômicas, a exceção de VEMG (Tabela 1). A contribuição deste efeito foi muito elevada na PROD $(82,8 \%)$, bem como nos indicadores de crescimento DHAS $(66,7 \%)$, ALT $(64,8 \%)$, duração do ciclo CIC $(54,7 \%)$ e emergência de plântulas EMG (48,0\%) (Tabela1).

Também foi determinada influência significativa do efeito varietal $(V)$ em todas as variáveis agronômicas, a exceção de EMG (Tabela 1). Sua contribuição sobre a VEMG foi muito elevada (46,7\%), bem como na duração do ciclo $(30,8 \%)$. Sobre as variáveis agronômicas relacionadas ao crescimento seu efeito foi pronunciado em DCAP $(28,2 \%)$ e mais reduzido no restante DHAS $(14,9 \%)$ e ALT $(8,1 \%)$. A produção foi influenciada de forma significativa, mas pouco expressiva $(3,4 \%)$ (Tabela 1$)$.

Com relação à duração do ciclo, todas as cultivares apresentaram ciclo mais curto no segundo cultivo, a cultivar Rumbosol 91 apresentou ciclo mais longo comparado ao das demais cultivares em ambas as semeaduras (Tabela 2). Todas as cultivares apresentaram significativa redução de ciclo em relação ao obtido no centro-sul do Brasil (Castro et al.,1997), que indicam ciclos de 90 a 130 dias para cultivares precoces e tardias, respectivamente. A altura de todas as cultivares foi maior na primeira época de semeadura, a exceção das cultivares Agrobel 920 e Morgan 742 que apresentaram respostas equivalentes em ambas épocas. As alturas obtidas pela cultivar M 742 são

Tabela 1 - Valores de quadrado médio e componentes de variância para as variáveis agronômicas avaliadas em função do modelo de análise adotado.

\begin{tabular}{|c|c|c|c|c|c|c|c|c|c|c|c|c|c|}
\hline \multirow[b]{2}{*}{$\overline{F V}$} & \multirow[b]{2}{*}{ g.l. } & \multicolumn{3}{|l|}{ EMG } & \multicolumn{3}{|l|}{ VEMG } & \multicolumn{3}{|l|}{$\overline{\mathrm{ALT}}$} & \multicolumn{3}{|c|}{ DHAS } \\
\hline & & $\mathrm{QM}$ & $p$ & $(\%)$ & $\mathrm{QM}$ & $p$ & $(\%)$ & $\mathrm{QM}$ & $p$ & $(\%)$ & $\mathrm{QM}$ & $p$ & $(\%)$ \\
\hline $\mathrm{E}$ & 1 & 3040,08 & $* *$ & 48,0 & 0,02 & n.s. & 0,0 & 11056,50 & $* *$ & 64,8 & 2,63 & $* *$ & 66,7 \\
\hline PROD & 5 & 39,35 & n.s. & 0,0 & 12,50 & $* *$ & 46,7 & 737,92 & $* *$ & 8,1 & 0,21 & $* *$ & 14,9 \\
\hline PROD & 5 & 319,23 & $* *$ & 27,7 & 1,36 & n.s. & 0,0 & 288,02 & n.s. & 4,8 & 0,02 & n.s. & 0,0 \\
\hline \multirow[t]{2}{*}{ PROD } & 5 & 319,23 & $* *$ & 27,7 & 1,36 & n.s. & 0,0 & 288,02 & n.s. & 4,8 & 0,02 & n.s. & 0,0 \\
\hline & \multicolumn{4}{|c|}{$\mathrm{CIC}$} & \multicolumn{3}{|c|}{ DCAP } & \multicolumn{3}{|c|}{ PROD } & & & \\
\hline FV & g.l. & $\mathrm{QM}$ & $p$ & $(\%)$ & $Q M$ & $p$ & $(\%)$ & QM & $p$ & $(\%)$ & & & \\
\hline $\bar{E}$ & 1 & 494,08 & $* *$ & 54,7 & 39,79 & $* *$ & 41,7 & 17141080,00 & $* *$ & 82,8 & & & \\
\hline V & 5 & 97,48 & $* *$ & 30,8 & 9,94 & $* *$ & 28,2 & 402066,16 & $* *$ & 3,4 & & & \\
\hline$E^{*} V$ & 5 & 5,88 & n.s. & 0,5 & 1,27 & n.s. & 1,0 & 171326,94 & n.s. & 2,1 & & & \\
\hline Erro & 36 & 5,19 & & 14,0 & 1,12 & & 29,1 & 99860,98 & & 11,7 & & & \\
\hline
\end{tabular}

*EMG - Emergência; VEMG - Velocidade de emergência; ALT - Altura das plantas; DHAS - Diâmetro da haste; CIC - Duração do ciclo; DCAP - Diâmetro do capítulo; PROD - Produtividade; $\mathrm{s}_{(\%)}$ - Componentes de variância relativo (\%); n.s. - não significativo; ${ }^{* *}$ - altamente significativo 
próximas das registradas em trabalho realizado por Gomes et al. (2003). Na primeira semeadura, a cultivar Rumbosol 91 apresentou valores superiores, enquanto que na segunda todas as cultivares foram equivalentes (Tabela 2).

Quanto ao diâmetro da haste, todas as cultivares apresentaram valores superiores na primeira época, sendo que Rumbosol 91 foi superior em ambas semeaduras (Tabela 2). Já quanto ao diâmetro do capítulo, todas as cultivares foram equivalentes em ambas semeaduras a exceção de C 11 e M 742 que apresentaram, na primeira época de semeadura, valores superiores; na primeira semeadura, a cultivar $\mathrm{C} 11$ apresentou os maiores valores de diâmetro do capítulo, enquanto que na segunda as cultivares AG 910 e RUMB 91 o apresentaram (Tabela 2).

Os valores médios de diâmetro de capítulos obtidos neste trabalho estão dentro da ampla variação $(6$ a $50 \mathrm{~cm})$ indicada para a cultura por Frank \& Szabo (1989). Diâmetros de capítulos muito superiores a $17 \mathrm{~cm}$ produzem menor quantidade de grãos cheios, resultando em menor produção (CETIOM, 1983). Por outro lado, capítulos muito reduzidos indicam limitações no desenvolvimento com grande influência na produção (Jain et al., 1978). Em relação a cultivar M 742, os valores obtidos nas duas épocas $(15,9$ e 13,9 cm) são semelhantes aos obtidos em trabalho realizado por Gomes et al. (2003) em São Paulo, objetivando avaliar o efeito do estresse hídrico sobre a altura e o diâmetro dos capítulos.

Os valores de emergência de plântulas obtidos foram maiores no primeiro cultivo, para todas as cultivares, a exceção de AG 910, C 11 e M 742, em que as épocas foram equivalentes; na primeira semeadura, a emergência de todas as cultivares foi equivalente, enquanto que na segunda se destacou a cultivar Cargill 11 (Tabela 2 e Figura 1).

As velocidades de emergência foram equivalentes em ambas as épocas, sendo que AG 920 foi superior (Tabela 2). A produção foi superior em todas as cultivares na primeira semeadura, sendo que nesta semeadura todas as cultivares foram equivalentes em produtividade, entretanto na segunda época de semeadura as cultivares AG 910 e RUMB 91 foram superiores (Tabela 2 e Figura 2). As produtividades médias obtidas, principalmente na primeira época de semeadura $\left(E_{1}\right)$ são superiores a média nacional que está em torno de $1500 \mathrm{~kg}$.ha${ }^{1}$. Estes valores de produtividade, segundo as indicações de Castro et al. (1997) quanto a custos e receita total, resultariam em rentabilidade, tendo em vista que são superiores aos 1333 kg.ha ${ }^{-1}$ necessários para acobrir o custo variável.

Ressalta-se a maior duração do ciclo de RUMB 91 (81-84 dias), na primeira semeadura em relação a duração do ciclo de C 11 (71-81 dias), no mesmo período (Figura 2). Esta cultivar apresenta ciclo mais longo em relação aos demais em estudo. Foi obtida maior taxa de emergência de RUMB 91 (67-91\%), no primeiro cultivo em relação a taxa de C 11 (52-77\%), no mesmo período (Figura 2).

Os teores médios de óleo obtidos para as cultivares em estudo estiveram ao redor de $37 \%$ e $41 \%$, respectivamente para o primeiro e segundo cultivos de girassol em Roraima. Estes valores estão dentro da amplitude $(10 \mathrm{a} 60 \%)$ de valores indicados por Frank \& Szabo (1989) como os esperados para a cultura do girassol, apresentando variações quanto aos materiais avaliados.

Tabela 2 - Valores médios das variáveis agronômicas em função das cultivares e época de cultivo, ordenados segundo o teste de Tukey $(\mathrm{a}=0,05)$.

\begin{tabular}{|c|c|c|c|c|c|c|c|c|c|c|c|c|c|c|c|c|}
\hline \multirow[b]{2}{*}{ Cultivares } & \multicolumn{4}{|c|}{ EMG } & \multicolumn{4}{|c|}{ VEMG } & \multicolumn{4}{|c|}{ ALT } & \multicolumn{4}{|c|}{ DHAS } \\
\hline & E1 & & E2 & & E1 & & E2 & & E1 & & E2 & & E1 & & E2 & \\
\hline AG 910 & 64,8 & a1 & 56,8 & $a b 1$ & 7,5 & $a b 1$ & 7,6 & $a b 1$ & 174,7 & $a b 1$ & 138,3 & a2 & 2,6 & $a b 1$ & 2,1 & $a b 2$ \\
\hline AG 920 & 76,5 & $\mathrm{a} 1$ & 55,5 & $a b 2$ & 9,6 & a1 & 9,8 & $\mathrm{a} 1$ & 173,8 & $a b c 1$ & 141,0 & a1 & 2,7 & $a b 1$ & 2,1 & $a b 2$ \\
\hline C 11 & 64,8 & $\mathrm{a} 1$ & 62,0 & $\mathrm{a} 1$ & 5,9 & b1 & 6,8 & $a b 1$ & 162,8 & bc1 & 143,3 & $\mathrm{a} 2$ & 2,7 & $a b 1$ & 2,3 & $a b 2$ \\
\hline EMBR 122 & 73,8 & $\mathrm{a} 1$ & 48,0 & b2 & 8,2 & ab1 & 8,9 & $a b 1$ & 163,7 & bc1 & 138,3 & a2 & 2,4 & b1 & 2,0 & b2 \\
\hline M 742 & 62,3 & a1 & 57,8 & $a b 1$ & 7,2 & b1 & 6,9 & $a b 1$ & 146,5 & c1 & 128,5 & a1 & 2,6 & b1 & 2,0 & $a b 2$ \\
\hline \multirow[t]{2}{*}{ RUMB 91} & 79,3 & a1 & 45,8 & b2 & 7,5 & $a b 1$ & 6,1 & b1 & 191,6 & a1 & 141,8 & a2 & 2,9 & a1 & 2,4 & a2 \\
\hline & & & $\mathrm{CIC}$ & & & & CAP & & & & ROD & & & & & \\
\hline Cultivares & E1 & & E2 & & E1 & & E2 & & E1 & & E2 & & & & & \\
\hline AG 910 & 81,8 & b1 & 76,5 & b2 & 18,0 & $a b 1$ & 16,8 & a1 & 2859,5 & a1 & 1934,5 & $\mathrm{a} 2$ & & & & \\
\hline AG 920 & 81,8 & b1 & 75,0 & b2 & 17,0 & ab1 & 15,5 & $a b 1$ & 2403,8 & $\mathrm{a} 1$ & 1523,3 & $a b 2$ & & & & \\
\hline C 11 & 83,3 & b1 & 76,5 & b2 & 18,6 & a1 & 15,3 & $a b 2$ & 3064,3 & $\mathrm{a} 1$ & 1418,3 & $a b 2$ & & & & \\
\hline EMBR 122 & 80,5 & b1 & 75,0 & b2 & 15,9 & b1 & 14,4 & b1 & 2498,3 & a1 & 1294,3 & b2 & & & & \\
\hline M 742 & 81,8 & b1 & 77,0 & b1 & 15,9 & b1 & 13,9 & b2 & 2459,5 & a1 & 1352,0 & $a b 2$ & & & & \\
\hline RUMB 91 & 92,0 & $\mathrm{a} 1$ & 82,5 & a2 & 18,2 & $a b 1$ & 16,8 & a1 & 3022,5 & a1 & 1614,5 & a2 & & & & \\
\hline
\end{tabular}

*EMG - Emergência; VEMG - Velocidade de emergência; ALT - Altura das plantas; DHAS - Diâmetro da haste; DCAP - Diâmetro do capítulo; CIC - Duração do ciclo; PROD - Produtividade; $\mathrm{E}_{1}$ - cultivo de janeiro a abril; $\mathrm{E}_{2}$ - cultivo de julho a setembro. Letras na vertical - efeito das cultivares; Número na horizontal - efeito das épocas de cultivo 
(a)

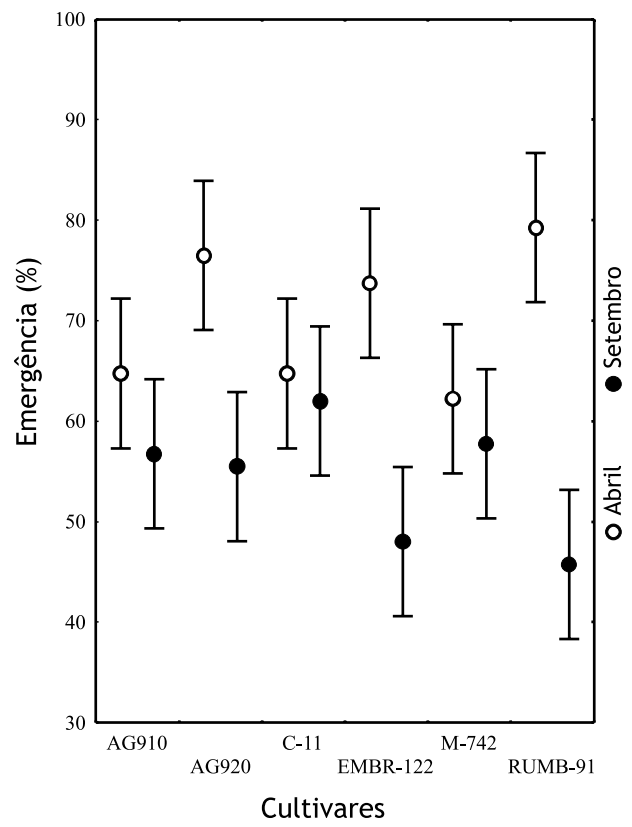

(b)

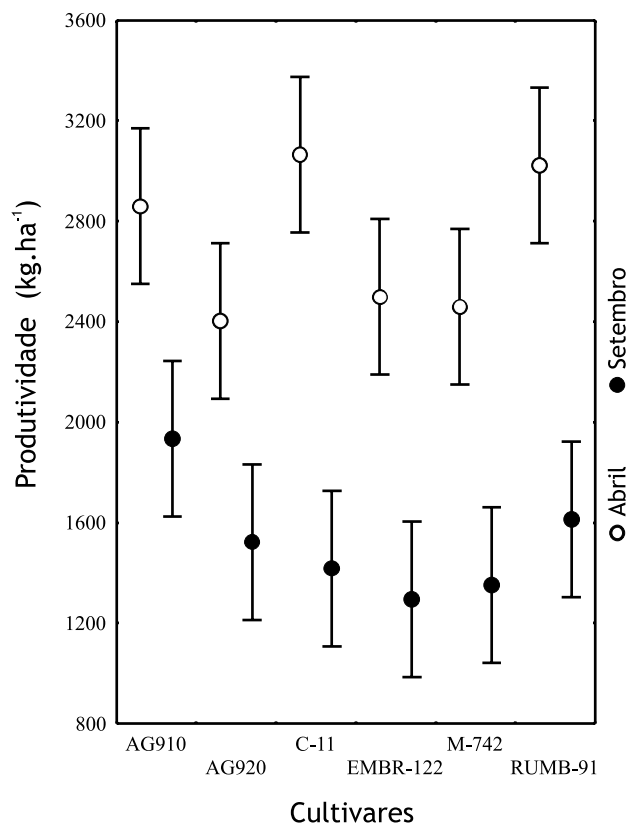

Figura 1 - Valores médios e intervalo de confiança de $95 \%$ de (a) emergência (\%) e (b) produtividade (kg.ha-1) em função das cultivares de girassol e época de cultivo

(a)

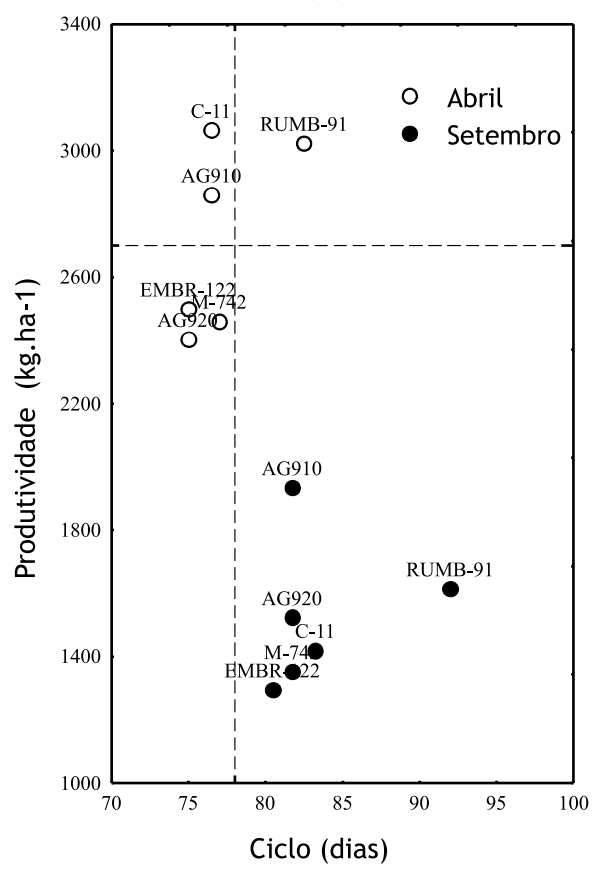

(b)

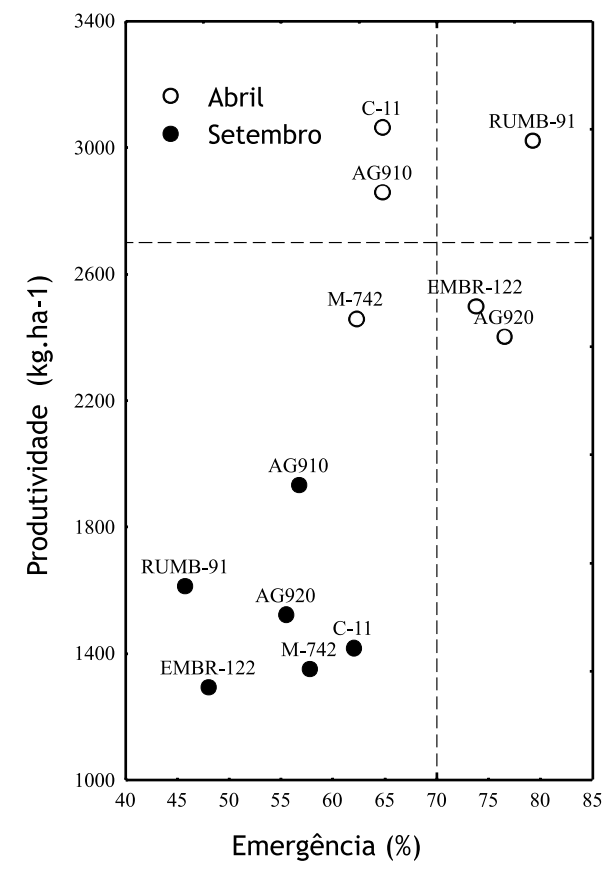

Figura 2 -Relação entre os valores médios de (a) duração do ciclo e (b) emergência (\%) em função da produtividade das cultivares avaliadas nas duas épocas de colheita. 
Pelos resultados obtidos, sugere-se a utilização das duas cultivares (Cargill 11 e Rumbosol 91) nas savanas de Roraima, otimizando-se o sistema de produção através da colheita inicial de Cargill 11.

\section{CONCLUSÕES}

Tomando-se como critério de decisão, relações entre a duração do ciclo, a taxa de emergência e a produtividade das cultivares, define-se que:as cultivares Cargill 11, Rumbosol 91 e Agrobel 910 foram as mais produtivas para cultivo nas condições climáticas das savanas de Roraima; e a semeadura em janeiro é mais indicada, comparada a de julho.

\section{BIBLIOGRAFIA CITADA}

Abreu, J.B.R. de; Menezes, J.B.O.X. de; Scofield, H.L.; Scolforo, L.; Araújo, L.A.; Souza, M.M.; Junior, É.P.do N.; Santos, A.P. 2001. Avaliação da produção de capítulos e de matéria seca total de quatro cultivares de girassol (Helianthus annuus). In: Reunião Nacional de Pesquisa de Girassol, 14. Simpósio Nacional Sobre a Cultura do Girassol, 2., 2001, Rio Verde. Resumos... Rio Verde: FESURV/IAM. 180 p. p.4849. (FESURV. Rvdocumentos, 1).

Bolson, E.L. 1990. Técnicas para a produção de sementes de girassol. EMBRAPA/ SPSB. Fev.

Castiglioni, V.B.R.; Balla, A.; Castro, C.de; Silveira, J.M. 1997. Fases de desenvolvimento da planta de girassol. Londrina: EMBRAPA CNPSo. 24p. (EMBRAPA-CNPSo. Documentos, 58).

Castro, C. de; Castiglioni, V.B.R.; Balla, A.; Leite, R.M.V.B. de C.; Melo, H.C.; Guedes, L.C.A.; Farias, J.R. 1997. A cultura do girassol. EMBRAPA/CNPSo. Londrina.36p. (Circular Técnica, 13).

CETIOM. 1983. Physiologie de la formation du rendement chez le tournesol. Information Techniques, v.83, p.3-72.

Costa, V.C.A. da; Silva, F.N. da; Ribeiro, M.C.C. 2000. Efeito de épocas de semeadura na germinação e desenvolvimento em girassol (Helianthus annuus L.). Revista Científica Rural. 5(1):154-158.

EMBRAPA, Centro Nacional de Pesquisa de Soja. 1999. Informes da avaliação de genótipos de girassol, 1998. Londrina, 92 p. (EMBRAPA-CNPSo. Documentos, 21).

EMBRAPA, Centro Nacional de Pesquisa de Soja. 1983. Indicações técnicas para o cultivo do girassol. Londrina, $40 \mathrm{p}$. (Documentos, 3).

Endres, V.C. 1993. Avaliação de cultivares de girassol no Mato Grosso do Sul 1991/92. In: Reunião Nacional do Girassol. 10, 1993. Goiânia. Anais... Goiânia: IAC, v.1, p.35-36.

Frank, J.; Szabo, L. A napraforgo Helianthus annuus, L. Budapest: Akadémiai Kiadó, 1989. 178p.

Gomes, E.M.; Ungaro, M.R.G.; Vieira, D.B. Influência da suplementação hídrica na altura de planta, diâmetro de capítulo, peso de sementes e produção de grãos. In: Reunião Nacional de Girassol, 15. Resumos... Ribeirão Preto, SP, 2003. 4p. CD-Rom

Jain, K.K.; Vaish, D.P.; Gupta, H.K.; Mathur, S.S. 1978. Studies on bollow seednes in sunflower. In: International Sunflower Conference, 8, Minneapolis, USA, 1978. International Sunflower Association. p.138-147.

Popinigis, F. 1985. Fisiologia da semente. Brasília, 2.ed.. 289p.

Reyes, F.G.R.; Garibay, C.B.; Húngaro, M.R.G.; Toledo, M.C.F. 1985. Girassol: cultura e aspectos químicos, nutricionais e tecnológicos. Campinas: Cargill. 88p.

Schneiter, A.A.; Miller, J.F. 1981. Description of sunflower growth stages. Crop Science, Madison, 21: 901-903.

RECEBIDO EM 13/08/2003
ACEITO EM 20/07/2005 